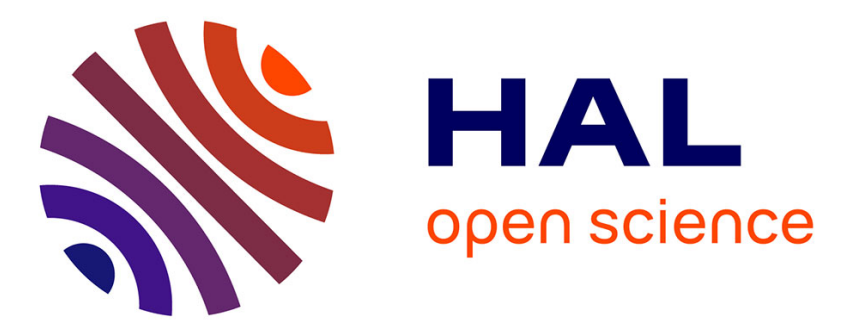

\title{
Body awareness disorders: dissociations between body-related visual and somatosensory information
}

Laure Pisella, Laurence Havé, Yves Rossetti

\section{To cite this version:}

Laure Pisella, Laurence Havé, Yves Rossetti. Body awareness disorders: dissociations between bodyrelated visual and somatosensory information. Brain - A Journal of Neurology , 2019, 142 (8), pp.21702173. 10.1093/brain/awz187 . hal-02346581

\section{HAL Id: hal-02346581 \\ https://hal.science/hal-02346581}

Submitted on 7 Nov 2019

HAL is a multi-disciplinary open access archive for the deposit and dissemination of scientific research documents, whether they are published or not. The documents may come from teaching and research institutions in France or abroad, or from public or private research centers.
L'archive ouverte pluridisciplinaire HAL, est destinée au dépôt et à la diffusion de documents scientifiques de niveau recherche, publiés ou non, émanant des établissements d'enseignement et de recherche français ou étrangers, des laboratoires publics ou privés. 


\title{
BADs: dissociations between body-related visual and somatosensory information
}

\author{
L. Pisella1, L. Havé 1,3 \& Y. Rossetti ${ }_{1,2}$ \\ 1 ImpAct Team, Lyon Neuroscience Research Center CRNL, INSERM U1028, CNRS UMR5292 and University Claude Bernard Lyon I, Villeurbanne, \\ France \\ 2 Plate-forme Mouvement et Handicap, Hospices Civils de Lyon, Centre de Recherche en Neurosciences de Lyon, 69500 Bron, France \\ 3 Hôpital d'instruction des armées Desgenettes, 69275 Lyon, France
}

\section{Glossary:}

Precuneus: medial part of the posterior parietal cortex, between the occipital (cuneus) and the anterior parietal (paracentral lobule) cortices, well located for visualsomatosensory integration.

Body image typically depicts mental representation of one's own body, arising from all sources of sensory and cognitive information, whereas body schema is used to depict the unconscious use of sensory information required by our motor system to maintain body posture and produce accurate movements. Brain lesions may result in deficits for either or both types of body representations.

Autotopoagnosia traditionally refers to patients who are impaired at pointing their body parts named by the experimenter. In addition, Herbet et al. describe a tactile version of autotopoagnosia, where patients are asked to name unseen body parts that receive a tactile stimulation. This deficit does not necessarily apply to others' body parts (heterotopoagnosia).

Motor neglect has been defined as a peculiar manifestation of unilateral neglect, whereby patients do not spontaneously use their controlesional limb in the absence of motor and somatosensory deficits. As for spatial neglect this condition is typically seen following right hemisphere lesions. For example a patient may struggle to take out a bag of tea from its wrapping with one hand even though being capable of doing it more easily with the two hands when instructed.

The Alien Hand syndrome refers to the deficit in recognizing a hand as one's own when its view is occluded. Herbet et al. describe a tactile version in which they apply tactile stimuli to the patient's unseen limb, and the patients do not feel that they are applied to their own body. The motor alien hand refers to the occurrence of purposeful movements that are unintended. When these movements are agonistic to the other hand, they are described as anarchic hand. Patients may also experience supernumerary limbs.

Fading limb corresponds to the subjective experience that when vision of the limb is removed, its position drifts and then its perception fades from consciousness.

Fig. 1 caption: Classification of body schema and body image disorders along the dimension proposed by Longo et al. (2010) (somatosensation corresponds to primary sensory processing, somatoperception corresponds to the localisation of somatic and postural stimuli subtended by parietal lobes, somatorepresentation corresponds to general semantic knowledge and body consciousness about arrangement and metric properties of body parts subtended by frontoparietal network) and schematic representation of their functional relationship (based on Rossetti et al. 2005 and Pitron et al. 2018) 
This scientific commentary refers to BRAIN-2019-00373.R1 'The antero-dorsal precuneal cortex supports specific aspects of bodily awareness', by Herbet et al. (doi:).

Information about our body arises from numerous origins: tactile, proprioceptive, nociceptive, vestibular, motor, cognitive and social. Only a limited portion of these are processed consciously and we remain unaware of many important processes necessary to maintain postural control or to undertake actions. Although its terminology and nosography are subjected to a high level of variability, body cognition is frequently viewed as a dyadic or triadic, that usually distinguish between the body schema and the body image (Longo et al. 2010; Pitron et al. 2018). Body awareness disorders (BADs) are uncommon in neurology. They may affect various levels of body cognition: odd localisation of body parts, distorted perception of body part, feeling of strangeness, loss of agency, reduced awareness of body parts, illusory feeling of movements or even supernumerary limbs. Typically following lesions of the right hemisphere, unilateral neglect may manifest itself in the body schema domain (hypokinesia or bradykinesia) as well as in the body image domain (e.g., tactile extinction or allochiria, personal neglect, anosognosia for hemiplegia, hemiasomatognosia, somatoparaphrenia). Microsomatognosia and macrosomatognosia may also result from lesions of the left hemisphere. Limb apraxia encountered following lesions of the left hemisphere may be related to body schema deficits. Following left posterior parietal lobe lesion leading to Gerstmann syndrome, both finger agnosia (inability to distinguish the fingers on the hand) and left-right disorientation, which may also concern body parts, may be classified as body image impairments. Anterior lesion of the left superior parietal lobule including the precuneus has been reported to cause illusory drift followed by fading limb in absence of vision (Wolpert et al 1998). Such body image impairment has not been reported in association with optic ataxia following posterior lesion of the left or right superior parietal lobule, whereas body schema deficits have been revealed in these latter patients who produce large localisation errors in a proprioceptive pointing task (Blangero et al 2007). Altogether, BADs are widely acknowledged to be associated with syndromes consecutive to parietal lobe lesions (Fig. 1) although they may follow other localizations but the reason of their presence or not remained mysterious. In this article of Brain, Herbert et al provide a possible answer to the scarcity of the BADs and their non-systematic association with the more classical parietal syndromes: BADs would be present only when the posterior parietal cortex lesion involves its medial part, i.e. the precuneus, and more specifically the antero-dorsal precuneus.

Neuroimaging data has involved the antero-dorsal precuneus in strikingly heterogeneous functions ranging from motor coordination, drawing and imitation to working memory and subject-verb integration (from Neurosynth online tool http://neurosynth.org/locations/6_-52_66_3/). In contrast, human brain lesions rarely affect this medial brain territory (in contrast to gliomas) explaining both why BADs after parietal stroke and specific neuropsychological reports of this region remain scarse. This highlights the originality and the functional relevance of this study of patients with glioma surgery that led the authors to propose that the antero-dorsal precuneus plays a crucial role in body awareness.

While circumscribed precuneus lesions are rather rare, the authors managed to assess 14 patients who underwent a surgery for glioma infiltrating the precuneus. Herbet et al. tested for a large number of neuropsychological functions prior to, early after (5 days) and late after (3 months) the surgery but the main aim of the study was to specifically assess BADs that were frequently observed in these patients, mostly at the acute stage. Seven of their patients showed BADs, which allowed the authors to functionally distinguish the antero-dorsal and the posterodorsal precuneal regions. In addition, they mapped in healthy controls the functional 
connectivity of these two anatomical seeds. They could establish that the resection cavity of the 7 patients showing BADs more precisely included the antero-dorsal part of the precuneus. The resting-state analysis revealed that this antero-dorsal region is involved in a wide sensori-motor network, suggesting that body schema disorders may result in body image ones. There are indeed complex interactions between body schema and body image but the body schema seems to have some primacy over the body image (Rossetti et al. 2005; Pitron et al 2018). The most frequent BADs observed by Herbet et al were tactile alien hand, fading limb, motor neglect and tactile autotopoagnosia. Moreover, these symptoms were often associated (patients P2 and P11 present both tactile alien hand and fading limb, P6 presents motor neglect, tactile alien hand and autotopoagnosia, P14 shows tactile alien hand and autotopoagnosia). In the following paragraph, we will speculate about the underlying mechanisms of these typically precuneusrelated BADs.

When vision of the limb is available, its proprioceptive and visual localisations slightly differ but are integrated into an averaged position estimate. In absence of direct vision of the limb, this position estimate drifts toward the proprioceptive location. This body schema perturbation is revealed by the observation of a progressive movement drift repeatedly toward the same non-random location when an individual alternates pointing movements in-between two visual targets in the dark (Smeets et al. 2006). When a large location mismatch is experimentally induced between vision and proprioception in healthy subjects, it has been shown to be resolved by selective fading of the concerned limb (Hogendoorn et al. 2009) or to impair tactile perception on this limb (Folegatti et al. 2009). Reciprocally, while touch may not provide additional information about finger position in space, since fingertip tactile stimuli should remain independent from the postural configuration of the upper limb, additional tactile information nevertheless improves proprioceptive localisation (Mikula et al. 2018). This may be due to the fact that tactile and proprioceptive information are ultimately coded within the same population of posterior parietal neurons within high-level spatial representations. Anterodorsal precuneus damage may affect multisensory integration and specifically lead the visual and proprioceptive experiences of a limb to largely depart from each other. Consequences may be feelings of strangeness under vision of the limb or of illusory movement when vision of the limb is prevented inducing a large position estimate drift. If moreover the limb proprioceptive representation is low weighted, its perception when visual feedback is removed may fade and it may disappear not only from body image but also from body schema (fading limb and motor neglect, respectively). Such neurologically induced visual-proprioceptive mismatch also affects tactile perception on the concerned limb. If the proprioceptive limb representation is unstable, it may result in tactile autotopoagnosia. If it fades from body image, it may produce tactile alien hand.

When additional primary somatosensory deficit is present, as in patient P2 of Herbet et al. whose resection cavity extended to the paracentral lobule, macrosomatognosia may also be observed. We had the opportunity to follow a patient (IK) who exhibited sustained BADs several years following surgery for an extradural haemorrhage. These disorders notably included macrosomatognosia of the right upper limb, in association with tactile autotopoagnosia (without heterotopoagnosia) and tactile but also motor alien hand (which has also been reported after right precuneus damage: Darby et al. 2018). Interestingly his left parietal cortex lesion included the precuneus and also impaired the primary proprioceptive function in isolation. During tactile examination with a blindfold, IK exhibited fully normal thresholds but most often complain that he had no idea where the stimulus was applied. $\mathrm{He}$ 
would even sometime burst: "you cannot touch me here because it is not on my body!". During several years IK spontaneously reported the perception of 3 right upper limbs: a visual one, an imagined one and a somatosensory one. These 3 hands were more independent of each other during the night, when the patient was lying in his bed in the dark. Interestingly, IK also initially complained of hand levitation, i.e. his controlesional hand would raise in the air and remain floating until he looked at it. In the daily life, he could be surprised by the vision of his right hand grasping the mirror while driving his car, as if his hand was levitating devoid of any notion of agency. In other experimental conditions, the patient was asked to sit at a table, with the right hand hidden under the table. An examiner was sitting behind him, presenting his hand on the table, at the right of the patient. Within a few seconds IK's body cognition took ownership of alien hand despite the fact of knowing it was obviously impossible. In addition, stupor and distressing reactions from the patients were often observed when the examiner removed his arm from the table without having warned the patient. The patient was horrified or disgusted, each time he saw the alien right hand moving while his own remained hidden. In short, this patient was facing conflictual perceptions and representations at different levels (sensory and cognitive) in absence of vision of his own hand.

Contrary to the general idea that BADs are predominantly associated with neglect and right hemisphere damage, the literature, in line with Herbet et al. data, do not provide evidence for such hemispheric lateralisation. This is consistent with the idea that they result from body schema deficits which are usually not lateralised in one hemisphere (like optic ataxia). One might think that this absence of hemispheric lateralisation of the BADs in Herbet et al. may be related to the fact that the deficits exhibited by their patients are predominantly present at the acute post-operative stage. Indeed, in the case of neglect, acute studies often report that symptoms are as frequent following lesions of either hemisphere. It is only at the chronic stage that the right hemisphere specialisation is revealed. One may speculate that the same phenomenon applies to BADs. However two out of four patients presenting with BADs at three months post-surgery had left lesion in Herbet et al. and IK showed chronic BADs several years following a left hemispheric lesion. This suggests that BADs may not be lateralised even at the chronic stage. Unlike some overt impairments such as dressing apraxia, BADs may alter body cognition more implicitly and remain undetected. Following Herbet $e t$ $a l$. it is therefore recommended to researchers and clinicians to carefully interview and examine patients for BADs when the antero-dorsal precuneus in lesioned, irrespective of the hemispheric side.

\section{REFERENCES}

1. Blangero A, Ota H, Delporte L, Revol P, Vindras P, Rode G, Boisson D, Vighetto A, Rossetti Y, Pisella L. Optic ataxia is not only 'optic': impaired spatial integration of proprioceptive information. Neuroimage. 2007; 36 Suppl 2:T61-8.

2. Darby RR, Joutsa J, Burke MJ, Fox MD. Lesion network localization of free will. Proc Natl Acad Sci U S A. 2018 16; 115(42):10792-10797.

3. Folegatti A, de Vignemont F, Pavani F, Rossetti $Y$, Farnè A. Losing one's hand: visual-proprioceptive conflict affects touch perception. PLoS One. 2009; 4(9):e6920. doi: 10.1371/journal.pone.0006920.

4. Hogendoorn $\mathrm{H}$, Kammers MP, Carlson TA, Verstraten FA. Being in the dark about your hand: resolution of visuo-proprioceptive conflict by disowning visible limbs. Neuropsychologia. 2009; 47(13):2698-703

5. Longo MR, Azañón E, Haggard P. More than skin deep: body representation beyond primary somatosensory cortex. Neuropsychologia. 2010; 48(3):655-68. 
6. Mikula L, Sahnoun S, Pisella L, Blohm G, Khan AZ. Vibrotactile information improves proprioceptive reaching target localization. PLoS One. 2018; 6;13(7):e0199627.

7. Pitron V, Alsmith $A$, de Vignemont $F$. How do the body schema and the body image interact? Conscious Cogn. 2018; 65:352-358.

8. Rossetti Y, Rode G, and Farnè A (2005) "Implicit body representation in action: a neuropsychological approach". In: Body image and body schema (De Preester $\mathrm{H}$ and Knockaert V, eds.), Benjamins Amsterdam, p. 111-115.

9. Smeets JB, van den Dobbelsteen JJ, de Grave DD, van Beers RJ, Brenner E. Sensory integration does not lead to sensory calibration. Proc Natl Acad Sci U S A. 2006; 103(49):18781-6.

10. Wolpert DM, Goodbody SJ, Husain M. Maintaining internal representations: the role of the human superior parietal lobe. Nat Neurosci. 1998;1(6):529-33

The authors report no competing interests 